\title{
Le livre de raison de François de Tapie de Monteil, capitaine au régiment de Poitou (1661-1670)
}

\section{Robert Le Blant}

Volume 13, numéro 4, mars 1960

URI : https://id.erudit.org/iderudit/302009ar

DOI : https://doi.org/10.7202/302009ar

Aller au sommaire du numéro

Éditeur(s)

Institut d'histoire de l'Amérique française

ISSN

0035-2357 (imprimé)

1492-1383 (numérique)

Découvrir la revue

Citer ce document

Le Blant, R. (1960). Le livre de raison de François de Tapie de Monteil, capitaine au régiment de Poitou (1661-1670). Revue d'histoire de l'Amérique française, 13(4), 562-573. https://doi.org/10.7202/302009ar d'utilisation que vous pouvez consulter en ligne. 


\section{DOCUMENTS INÉDITS}

\section{LE LIVRE DE RAISON \\ DE FRANÇOIS DE TAPIE DE MONTEIL CAPITAINE AU RÉGIMENT DE POITOU}

(1661-1670)

\section{LE CAPITAINE FRANCCOIS DE TAPIE DE MONTEIL}

Le nom, les surnoms et la qualité de François de Tapie de Monteil ont attiré l'attention des érudits canadiens qui leur ont fait subir quelques fluctuations au cours des élans de plume, provoqués par les conséquences d'une expédition en NouvelleFrance du régiment de Carignan-Salières, renforcé de 4 compagnies tirées des régiments de Poitou, Chambellé, Broglie et Orléans.

S'agissant de l'histoire héroïque par excellence de la population canadienne dont l'existence était à ce point menacée en 1665 par les terribles Iroquois, qu'une intervention de troupes françaises importante s'avérait indispensable, des sources diverses ont été utilisées pour l'identification des glorieux militaires demeurés sur les rives du Saint-Laurent plus populaires que le baron de Lahontant. C'est ainsi que François I ${ }^{\text {er }}$ de Tapie de Monteil a été qualifié François de Montail de Clairvacq, capitaine au régiment de Poitou, ${ }^{1}$ de Monteuil, ${ }^{2}$ enfin François Tapie de Monteil et de Clairac, ${ }^{3}$ bien qu'il ne semble pas avoir porté habituellement ce second surnom. Cet officier appartenait à la famille agenaise des de Tapie dont les représentants portaient le surnom de Monteil ou Monteilh ou Monteils tiré d'un fief situé sur les villages de Saint-Jean-de-Turac et de Saint-Pierre-

1 B. Sulte, Mélanges historiques (Montréal, 1922), VIII: Régiment de Carignan, $8^{\circ} \mathrm{Pa} 206$ (8) à la Bib. Nat.

2 Jugements du Conseil Souverain de la Nouvelle-France (Québec, 1885), I: 458, LK 12 1370, idem.

3 Régis Roy et Malchelosse, Le Régiment de Carignan (Montréal, Ducharme, 1925), inconnu à la Bib. Nat., aimablement communiqué par M. Claude de Bonnault. Le Jeune, Dictionnaire Général du Canada, également inconnu à la Bib. Not. 
de-Clairac, ${ }^{4}$ cette dernière paroisse ayant subsisté comme commune du canton de Puymirol, arrondissement d'Agen. Son identité a pu être établie grâce aux renseignements sur sa parenté figurant dans son livre de Raison conservé aux Archives du Lot et Garonne, ${ }^{5}$ précisés à l'aide d'autres documents qui ont permis l'édification d'une généalogie de sa famille demeurée sommaire, mais suffisante pour résoudre le problème qui nous intéresse. Les indications données par le capitaine ne sont pas, cependant, d'une clarté limpide et les extraits de documents personnellement établis par l'excellente comtesse de Raymond qu'il nous a fallu utiliser, ne sont pas toujours d'une rigoureuse exactitude.

D'après son livre de Raison, Francois ${ }^{\text {er }}$ de Tapie de Monteil qui signait « de Monteil » avait un frère au séminaire et une sœur qu'il qualifia «ma sœur d'Arcet ». Le fief de Monteil ayant appartenu aux de Tapie durant tout le $17^{\mathrm{e}}$ siècle, le frère séminariste correspond avec François II de Tapie, fils de Pierre ${ }^{\text {er }}$ de Tapie et d'Isabeau d'Arcet, qui fut prêtre et curé de Lugaignac. La sœur « d'Arcet», au sens strict, aurait été une sœur du capitaine mariée à un sieur d'Arcet. On peut comprendre aussi qu'il s'agissait d'Isabeau d'Arcet elle-même qui aurait été, dans ce cas, la belle-sœur du capitaine ou une fille d'un premier lit d'Isabeau d'Arcet, bien qu'une telle personne eût porté le nom de son père et non celui de sa mère. Nous n'avons trouvé aucune trace d'une demoiselle de Tapie mariée avec un sieur d'Arcet, union qui n'irait d'ailleurs pas à l'encontre de l'identification que nous allons proposer. Nous ne pensons pas qu'on puisse retenir cette hypothèse car les demoiselles Tapie de Monteil contemporaines du capitaine semblent connues.

La possibilité de considérer l'officier comme un beau-frère d'Isabeau d'Arcet ne paraît pas devoir être acceptée par suite de présomptions tirées du texte du Livre de Raison. Le document indique en effet que le capitaine fit à son père en 1669 et 1671 différents prêts d'argent, notamment pour payer la dépense de son frère Tapie au séminaire, les tailles du fief de Monteil et d'une métairie située à l'Ile Barrade. Il était, par conséquent, le fils d'un seigneur de Monteil vivant en 1669 et pourvu d'un autre fils relativement jeune comme se trouvant au séminaire. Ce seigneur de Monteil ne pouvait être Auguste de Tapie, décédé avant le 3 juillet $1624^{\circ}$ dont le capitaine aurait été le fils s'il avait été le beau-frère d'Isabeau d'Arcet. On trouve, en effet, un hom-

\footnotetext{
4 C 2241 aux Archives départementales de la Gironde, 13 rue d'Aviaud à Bordeaux.

5 Fonds de Raymond 137, aux Archives départementales du Lot et Garonne à Agen.

o Passim. Généalogie de la famille Tapie de Monteil.
} 
mage de Rose de Sévin, veuve d'Auguste de Tapie, mère de Pierre de Tapie, pour la terre de Monteil en date du 26 août 1634, et Pierre de Tapie ayant épousé Isabeau d'Arcet au plus tard en 1636, aucune discontinuité, bien que la période soit longue, n'apparaît entre lui et le mari d'Isabeau d'Arcet qui décéda avant le 20 juillet 1694. D'autre part, la métairie de l'Ile Barrade semble être entrée dans la famille de Tapie postérieurement aux dénombrements fournis par Pierre $\mathrm{I}^{\text {er }}$ de Tapie dont le dernier est du 2 mars 1655 et qui n'y font aucune allusion. Elle paraît donc avoir été apportée par Isabeau d'Arcet qui la posséda seule après le décès de son mari, Pierre $I^{\text {er }}$ de Tapie. Nous ne pouvons dissimuler qu'une certaine obscurité subsiste en l'absence d'un document établissant une filiation précise sur le sens de l'expression «ma sœur d'Arcet», mais il n'est pas exclu de penser que l'officier désigna sa sœur sous un surnom tiré du nom d'une terre d'Arcet qui exista certainement, car un Pierre de Tapie s'intitula au $18^{\mathrm{e}}$ siècle «sieur d'Arcet » et signa même «Darcet de Monteils ».7 On trouve dans le livre de Raison un autre emploi de surnom avec une allusion à «mon frère de Fonnuges 》, lecture douteuse mais ne correspondant à aucun élément trouvé dans les actes de la famille de Tapie. Il apparaît done que le capitaine désigna ainsi un fils d'un premier lit de sa mère Isabeau d'Arcet. D'autres expressions semblent distinguer l'existence d'un demi-frère et d'une demi-sœur de l'auteur du livre de Raison car on y trouve «mon frère de Tapie» pour désigner le séminariste et «ma sœur » tout court, comme si l'écrivain avait voulu discriminer une sœur de Tapie de sa sœur d'Arcet. Quoi qu'il en soit, les présomptions sont suffisantes pour permettre d'indiquer que François $\mathrm{I}^{\text {er }}$ de Tapie de Monteil, vraisemblablement plus âgé que son frère Francois II, le séminariste, était fils de Pierre Ier de Tapie de Monteil et d'Isabeau d'Arcet, petit-fils d'Auguste de Tapie et de Rose de Sévin. Dans ces conditions, le capitaine avait 3 frères et 3 sœurs issus du mariage de ses parents qui, à l'exception de l'ecclésiastique, contractèrent des alliances dans la région d'Agen; malheureusement l'étude des actes les concernant n'a fourni aucune précision au sujet de François ${ }^{\text {er }}$ de Tapie.

Ce dernier était à Paris, le 10 décembre 1661, et lieutenant commandant la compagnie de Lahais du régiment de Poitou en garnison à Blaye, le 20 décembre 1663, lorsqu'il partit avec son unité pour La Rochelle où le Roi le fit loger pendant un mois. Il y reçut, le 2 février 1664, l'ordre d'embarquer sur le vaisseau Le Brézé, très grand bâtiment de 1200 tonneaux d'après le capitaine,

7 Fonds de Raymond 72, fo. 339, aux Archives départementales du Lot et Garonne. 
monté par le lieutenant-général de Tracy avec 700 hommes à destination des Indes de l'Amérique méridionale. Son chef direct n'ayant pas voulu courir les risques du voyage, François de Tapie fut au nombre des braves qui acceptèrent un avancement périlleux et se vit nommer capitaine en titre le 22 février suivant. L'expédition commença par débarquer à Cayenne pour en expulser les Hollandais et remettre la colonie à la Compagnie des Indes occidentales, puis elle fit un séjour à La Martinique où les habitants s'opposaient par affection envers la famille Dyel au retour en France d'un des fils de du Parquet, c'est-à-dire, selon toute vraisemblance, Jean-Jacques Dyel dit d'Esnambuc, nommé lieutenant-général à La Martinique le 20 mars 1651 par son père, Jacques Dyel, dit du Parquet. Tracy imposa ce retour en France afin d'éviter toute éventualité d'une prise d'armes contre le nouveau gouverneur Clodoré. Il s'installa ensuite à La Guadeloupe dont les habitants étaient, au contraire, soulevés contre le gouverneur Houel qui pratiquait des extorsions de fonds à leur encontre. Le lieutenant-général lui décerna une lettre de cachet pour le contraindre à rendre le Fort-Saint-Louis et à partir par le premier bâtiment rendre compte de sa conduite au roi. Houel s'exécuta, s'embarqua sur un navire de guerre nommé Le Terron et le roi le remplaça par du Lyon, gentilhomme bourguignon dont Tapie de Monteil fit l'éloge. L'expédition resta 14 mois dans l'île parce que, d'après l'auteur du livre de Raison, elle jouissait d'un climat sain et n'était pas, comme les autres Antilles, pourvue de couleuvres qui attaquaient les habitants jusque dans leur lit. Ce séjour aussi agréable que paisible prit fin par un ordre de Louis XIV du 15 avril 1666, expédiant 4 compagnies au Canada, pour réduire les sauvages à l'obéissance, formule établissant de nos jours qu'on ne se faisait pas, alors, à la Cour, une idée très exacte de ce que pouvaient être les 5 nations iroquoises. La traversée fut heureuse et se termina la veille de la Saint-Jean, tandis que Salières, avec 8 compagnies de 50 hommes sur les 20 composant son régiment, atteignit Québec seulement le 17 août 1666. De Tapie de Monteil n'indique pas quelle part il prit aux travaux de fortifications qu'on commença par effectuer, mais seulement qu'il partit avec le gouverneur du Canada, de Courcelles, au mois de janvier, pour tenter de surprendre un village des Aniers par un froid terrible qui faisait mourir en marchant les hommes et les guides. La perte de ces derniers entraîna un échec sur lequel le capitaine s'étend assez peu. Il s'agit d'une erreur d'orientation qui entraîna les Français vers les établissements hollandais. De Tapie resta au Canada jusqu'au 5 novembre 1668, date à laquelle il prit la mer pour revenir à La Rochelle, le 29. Incorporé au régiment de Champagne, en garnison à Saint-Jean-Pied-de-Port 
dans la cie de Dézé, il arriva avec elle, le 12 octobre 1669, à Bordeaux, au Château-Trompette où un service d'un semestre sur deux lui permit de venir à Agen le Ier février 1670, d'aller à Cadillac, de revenir au Château-Trompette, le 2 juillet. Il prit ses repas à l'auberge de Bordeaux jusqu'à la fin d'août, date à laquelle il paya sa note, fit un compte de ses avances pour sa compagnie le 18 septembre, reçut du trésorier extraordinaire des guerres, Esmale, 187 livres le 27 octobre 1670, prêta 9 louis d'or à son père le 14 avril 1671. Nous ignorons ce que devint ensuite le capitaine François de Tapie de Monteil.

\section{LE MANUSCRIT DU LIVRE DE RAISON}

Le manuscrit $137 \mathrm{du}$ fonds de Raymond est un carnet en parchemin, formant porte-feuille non folioté, de $15 \mathrm{~cm}$. de haut sur 9 de large comportant un brochage assuré par des bouts de lacets aussi en parchemin dont l'ensemble est rompu. La plus grande partie du texte transcrit à l'encre est souvent passée et même effacée. Des restes d'inscriptions au crayon sont illisibles. L'écriture, lorsqu'elle a été conservée dans une mesure suffisante, est d'une lecture souvent d'autant plus difficile que l'orthographe a subi des variations continuelles, tandis que certains mots sont teintés d'accent gascon. P. Tamizey de Larroque a considéré le document comme un journal de voyage et récit d'expédition plutôt que comme un livre de raison, ${ }^{8}$ mais l'ensemble du manuscrit constitue bien un livre de raison, rubrique sous laquelle il a été classé dans le catalogue du fonds de Raymond par G. Tholin, archiviste départemental du Lot-et-Garonne. ${ }^{9}$

Un résumé sommaire de la croisière effectuée sous les ordres d'Alexandre de Prouville dit de Tracy ne peut être confondu avec un journal de voyage, et quelques lignes consacrées à une offensive contre les sauvages Aniers ne peut constituer un récit d'expédition. Ces indications sont en réalité noyées dans un ensemble assez confus dont l'objet n'est assurément pas celui d'être utile aux historiens, mais de constituer un aide-mémoire. C'est à des fins très diverses que l'auteur a pris note des événements et des idées dont il désirait conserver la trace. Une énumération des possessions anglaises et françaises en Amérique du Nord ne tend qu'à des buts très sommaires d'intelligence de la géographie car

8 Deux livres de Raison de l'Agenais suivis d'extraits d'autres registres domestiques et d'une liste récapitulative des livres de Raison publiés on inédits (Auch, Cocharaux et Paris, Alphonse Picard, 1893), 135, $8^{\circ} \mathrm{Lm}^{2} 301$, à la Bib. Nat.

${ }_{9}$ Catalogue des travaux personnels, dossiers généalogiques etc. de Mme la comtesse de Raymond (Agen, 1889), $8^{\circ} \mathrm{Q} 1395$. 
elle est dépourvue de toute prétention à l'inédit. Une citation de 4 vers, 2 dizains et 2 alexandrins corrects, certainement tirés de l'œuvre d'un auteur littéraire, tendent cependant à la réalisation d'un intérêt pratique pour le copiste, s'agissant de mettre fin à une situation rendue pénible par les inconvénients d'une incertitude amoureuse dont le capitaine connaissait l'objet mieux que nous. Les fins utilitaires s'avèrent aussi, en dépit d'une certaine originalité avec la reproduction de recettes contre des affections alors fréquentes aux Antilles, la colique, la peste et la fièvre. Les remèdes considérés comme susceptibles de procurer la guérison de cette dernière maladie sont présentés sous la forme d'une alternative constituée par l'utilisation d'un chardon mis en poudre ou l'emploi d'une torche attachée au col qu'on laisse pendre sur l'estomac... De nos jours, la chaleur paraîtrait plus indiquée contre la colique. Du reste, les intentions pratiques dominent incontestablement par la présentation de l'ensemble du manuscrit composé surtout d'inscriptions comptables. Elles révèlent un grand souci d'exactitude triomphant de certaines naïvetés: certains paiements ayant le caractère d'avances consenties lors de la nomination d'officiers subalternes sont revêtus de la signature de François de Tapie, utilisant son surnom de Monteil et appliquant une règle à lui toute personnelle par application de laquelle la réalité des créances était constatée par leur bénéficiaire agissant unilatéralement.

Les mentions d'opérations comptables sont soigneusement séparées par un espace blanc permettant de discriminer les actes les uns des autres et cette présentation semble devoir être respectée par une publication. De nombreuses inscriptions de paiements, de dettes et de prêts ont été cancellées après règlement suivant un usage suivi par les notaires de l'époque, mais avec mention toutefois par les tabellions des motifs de l'annulation. L'auteur indique quelquefois seulement le solde de l'opération restant à régler et ce procédé qui facilite les déductions confirme bien le caractère général d'aide-mémoire qu'il faut attribuer au livre de Raison. Cela n'explique guère, par contre, que son étude soit rendue difficile par l'absence de tout ordre chronologique, imperfection pouvant résulter d'une fantaisie apportée dans la reliure ou d'une composition à l'aide de notes recopiées pêle-mêle. L'imbroglio qui est presqu'effarant commence par une énumération géographique, continue par des détails portant sur l'équipement des troupes au Canada depuis 1665 jusqu'au retour de 1668, revient au récit du départ pour Cayenne en 1664, suivi du récit du voyage et du séjour au Canada. Après des indications sur la vie de garnison à Bordeaux, l'ouvrage revient encore au séjour en Canada, puis nous ramène à Bordeaux en 1670 . 


\section{PRINCIPALES INDICATIONS HISTORIQUES DONNEEES PAR LE LIVRE DE RAISON}

La partie du manuscrit qui est directement la plus intéressante est certainement constituée par les indications malheureusement très brèves données sur la croisière faite sous les ordres d'Alexandre de Prouville dit de Tracy, depuis le printemps de 1664 jusqu'à l'automne de 1668, à Cayenne, la Martinique, la Guadeloupe et le Canada. Tapie de Monteil donne des précisions inédites particulièrement importantes pour l'expédition dirigée par de Courcelles contre la tribu iroquoise des Aniers en janvier 1666. Le livre de Raison est à notre connaissance le seul document précisant la lourdeur des pertes françaises qui s'élevèrent à 400 hommes et de celles des sauvages ennemis qui semblent avoir subi certains chocs en rase campagne. Les documents de l'époque sont sur cette affaire assez discrets, certainement à tort en ce qui concerne les pertes ennemies dont l'intensité peut expliquer des conversations pacifiques postérieures. Leur origine demeurerait autrement assez confuse. Les renseignements les plus abondants sont ceux qui sont donnés sur la vie militaire en France et au Canada à une époque qui n'est pas encore celle de Frontenac. De Tapie a noté très souvent des opérations comptables issues de ses fonctions, notamment les avances consenties aux officiers subalternes et aux soldats, la perception des sommes et des effets destinés à sa compagnie, l'encaissement de ses appointements, mais elles ne sont pas exclusives de dépenses et recettes de la vie courante présentant quelquefois un caractère assez inattendu. Les tractations entre militaires étaient compliquées: il est certain que les officiers touchaient une somme assez importante le jour de leur promotion. De Canchy toucha 160 livres lorsqu'il fut nommé enseigne, de La Fontaine 100 écus lorsqu'il fut nommé lieutenant. Il apparaît bien qu'il s'agissait d'avances sur la solde car cette précision est donnée pour le versement fait à de La Fontaine, mais les opérations financières effectuées entre le capitaine et les officiers placés sous ses ordres débordaient sur les affaires privées. L'enseigne de Lérolle ne toucha pas entièrement sa solde et de Tapie nota qu'il fallait tenir compte de perceptions de vivres pendant un an et d'eau-de-vie appartenant au capitaine personnellement. L'enseigne de Lafont lui emprunta 450 livres remboursables à la merci du capitaine, pour acheter le drapeau qu'il portait dans la compagnie au .Canada, mais aussi 20 livres pour acheter de l'eau-de-vie et 4 livres pour l'acquisition de barils. 
Les deux hommes étaient en affaires depuis longtemps, car, à Paris, le 10 décembre 1661, Tapie avait vendu à crédit un cheval à Lafont qui lui emprunta, par surcroît 15 livres destinées à payer sa dépense après son départ de Paris. L'enseigne remboursa par fractions notamment de 120 livres, monnaie du Canada, de 10 livres sans autre précision et d'une aune et demie de tabis blanc, étoffe que nous n'avons pu identifier. De Lérolle emprunta 22 livres monnaie de France mais de Tapie fut débiteur envers lui pour un montant de 33 livres 10 sols, monnaie de Canada, dont la cause n'est pas indiquée. Les motifs d'une retenue de 4 sols par prêt au soldat Bourguignon et d'une autre de 3 sols au soldat La Roche demeurent inconnus. Le livre de Raison indique, tantôt d'une façon assez aléatoire, tantôt avec la plus grande précision, certains mouvements d'officiers dans la compagnie. Ils commencèrent le 24 février 1664 à bord du vaisseau Le Brézé, par la promotion de La Fontaine comme lieutenant et celle de Canchy comme enseigne. Il s'agit sans doute de la suite du mouvement déclenché par l'avancement du lieutenant de Tapie. De Lérolle qui fut reçu enseigne le 17 avril 1765 à La Guadeloupe n'était donc pas le même personnage que de Canchy avec qui les historiens ont l'habitude de le confondre. Promu lieutenant le 29 mai 1666 au Canada, de Lérolle fut enlevé par les Iroquois durant une partie de chasse au lac Champlain. Le journal de Salières nous apprend qu'il fut libéré. De Lafont, parti simple soldat, fut nommé enseigne portant le drapeau, le 29 mai 1666, vraisemblablement à la place de Lérolle promu lieutenant le même jour, une inscription portant la date du 21 mai ayant été cancellée, mais la précision du remplacement n'est pas donnée. De Canchy et de La Fontaine, ce dernier probablement remplacé par de Lérolle, n'apparaissent plus, ce qui est vraiment dommage, étant donné les affectations quelque peu arbitraires effectuées par les historiens canadiens au bénéfice de leurs chers militaires. De Lafont dont le nom est souvent dépourvu de « $t$ » dans le livre de Raison a été considéré jusqu'à présent comme s'appelant Rolland de Lafons, enseigne de la compagnie de La Tour, le Père Le Jeune ayant emboîté le pas plus ou moins bien cadencé de Régis Roy et Malchelosse sur ce point.

Il suffit d'avoir conservé le souvenir d'une exposition d'histoire coloniale organisée à Paris où figurait l'invraisemblable reproduction, grandeur nature, d'un soldat du régiment de Carignan-Salières déguisé en coureur de bois, pour comprendre l'intérêt des détails précis donnés par le capitaine sur l'habillement et l'équipement de sa compagnie au Canada. Ses hommes étaient vêtus en commençant par le haut d'un chapeau à ruban, pouvant être remplacé par un bonnet, d'une cravate, d'une chemise, d'un 
justaucorps ou gilet, d'un habit de drap, d'un haut-de-chausses, d'une paire de bas de rame, d'une paire de souliers, d'un capot ou manteau. On trouve une mention de 15 pelliques ou manteaux de fourrures constituant une adjonction fort vraisemblable aux uniformes. Munis de couvertures et de peignes, instruments dont l'existence paraît confirmer qu'ils ne portaient pas de perruques, les soldats réparaient leurs vêtements avec des aiguilles, du fil et des ciseaux. Ils avaient des chaudières. On ne trouve aucune mention de cuillers, de fourchettes ni de gamelles, mais l'énumération des objets relevés ne paraît pas devoir être considérée comme restrictive, car les soldats devaient avoir des sacs dont il n'est pas plus question que de fusils ou mousquets qu'ils portaient certainement. Certaines omissions peuvent s'expliquer du fait qu'il s'agissait d'objets ne donnant pas lieu à remplacement habituel. En dépit des imprécisions concernant le nombre des hommes bénéficiant des distributions, il est permis de penser que chaque soldat avait 2 habits de drap, car ce vêtement est toujours indiqué par paires, de même la consommation de ruban était assez importante car on comptait par 30 et 50 aunes. Les paires de ciseaux n'étaient pas individuelles, mais chacun avait probablement 2 aiguilles.

Les indications données sur le pouvoir d'achat et le coût de la vie d'un officier au Canada sont assez vagues et n'interviennent qu'à l'occasion des affaires personnelles du capitaine avec une précision suffisante, car s'il reçut 1100 et quelques livres argent de France pour ses appointements de 1666, cette somme comprit la valeur de denrées accordées par le roi et rachetées par un certain Beguain qui paraît avoir rempli des fonctions officielles. De Tapie régla en monnaie d'or sa pension à sa logeuse, mademoiselle Amiot, qui reçut la somme paraissant considérable de 49 louis pour la période probable du 10 septembre 1667 au 5 novembre 1668 , la mention comptable semblant avoir été celle d'un total. L'hôtesse canadienne lui acheta une barrique d'eaude-vie pour 40 écus, monnaie de Canada; l'officier vendit un fusil à une demoiselle de La Tillerie pour 20 livres, aussi monnaie de la colonie, une barrique de vin à un certain Queribon pour 10 livres de la même monnaie, mais une opération portant sur des couteaux fut plus compliquée: de Tapie vendit 2 grosses, c'est-àdire 2 fois 12 douzaines de couteaux 28 livres la grosse, monnaie de France, moyennant un billet payé ou plus vraisemblablement payable en peaux d'orignaux; ce mode de règlement ne saurait surprendre, car l'acquéreur fut le fameux marchand Jean Péré, que nous avons pu identifier. Né à Arthez, en Béarn, fils de Gérard Péré, marchand et d'Arosette de Bosaussy, il épousa à 
La Rochelle, suivant contrat du 23 avril 1669, passé devant le notaire Teuleron, ${ }^{10}$ Marie Bonneau, native de La Rochelle, fille de feu François Bonnaud (sic), marchand de La Rochelle et de Catherine Nolleau. Jean Péré fut assisté par son frère, Arnaud, qui avait épousé dans la même ville, suivant contrat du 31 juillet 1661, Suzanne Grignon, fille d'Antoine Grignon et de Suzanne Suppet. ${ }^{11}$ Arnaud Péré acheta 2 grandes maisons dans la paroisse Saint-Jean-du-Perrot, la première Grande-Rue, suivant contrat du 26 mars 1669, ${ }^{12}$ la seconde, donnant également par devant sur la Grande-Rue, mais située rue Saint-Jean du Perrot, suivant contrat du 7 mars $1671 .{ }^{13}$ Nos identifications sont certaines, en dépit des signatures «Pérez ».

Pour en revenir à l'opération sur 2 grosses de couteaux et par son effet, le prix de la douzaine de couteaux était d'un peu plus de 2 livres, mais de Tapie sut réussir une autre vente d'instruments tranchants au prix de 3 livres la douzaine, sans qu'on sache s'il s'agissait de monnaie de France ou de monnaie de Canada. Dans la première hypothèse, qui paraît vraisemblable à défaut d'indication contraire, le capitaine aurait consenti une réduction pour le prix de gros, à moins que son commerce n'aît porté sur plusieurs sortes de couteaux. Les barriques devaient avoir des capacités très différentes si certains vins n'atteignaient pas des prix très élevés, car la blanchisseuse du capitaine, madame Lapointe, lui acheta, en 1665, 1 barrique de vin pour 70 livres, monnaie de Canada. Les marchands du pays étaient assez bien pourvus, car de Tapie put acquérir des bas de rame noire et un baudrier en s'adressant à un certain Viail. Il trouva un tailleur nommé La Rose et sa blanchisseuse, madame Lapointe, s'acquittait de son office pour une somme forfaitaire de 5 livres par mois, sans précision permettant de savoir s'il s'agissait d'argent de France ou d'argent de Canada, mais avec livraison à domicile, car certains paiements furent effectués à son «garson». En France, une demoiselle Cinqréze, bordelaise dont le prénom était Jolie, fit faire pour 42 écus blancs, soit 126 livres de linge au capitaine. Celui-ci prenait pension pour ses repas à l'auberge de Baladon alias Taladon, à Bordeaux, pour 15 sols par jour le 2 juillet 1670 et 20 sols par jour le mois d'août suivant. Il était de service effectif seulement un semestre sur deux au ChâteauTrompette où un tapissier nommé maître Arneau lui installa un

10 Registre de 1669, à la date, aux Arch. Dép. de la Charente Maritime. Communication de M. Marcel Delafosse, directeur.

11 Manuscrit 1850, minutes de Moreau, 1661, à la date, à la Bibliothèque municipale de La Rochelle.

12 Registre de Teuleron, 1669 , déjà cité, fo. 62.

13 Idem, registre de 1671 , fo. 31 , vo. 
lit en location pour $1 / 2$ pistole, soit 5 livres par mois, parce qu'il s'agissait d'un «cardier», expression paraissant indiquer le confort d'un matelas en laine cardée. Ce couchage paraît avoir été assez cher, puisqu'il représentait 3 sols par jour. La subsistance de la Compagnie représentait 450 livres par mois du 19 août au 30 septembre 1670 , ce qui, même en comptant seulement un effectif de 30 hommes, faisait 11 livres par mois et 7 sols par jour pour un soldat. Un effectif de 50 hommes ferait tomber ce prix de revient à 4 sols $1 / 2$ par jour. Un justaucorps, c'est-à-dire un gilet de « baraguan » avec de la dentelle de Valenciennes et 6 aunes de doublure, coûtait 27 livres en 1669 et on avait alors plusieurs perruques pour 14 livres. Suivant une contre-partie devant être normale en principe, de Tapie toucha 150 livres pour ses appointements de mai et juin 1670, 75 livres pour le mois de juillet 1670, 74 livres pour le mois d'août suivant. Il gagnait donc par jour 2 livres $1 / 2$ dont sa nourriture représentait les $2 / 5$ et le louage de lit le 1/15 lui laissant 40 livres par mois, soit 1 livre 1/3 par jour avec l'obligation de s'habiller et de se faire blanchir. La grosse dépense de 126 livres pour la confection de linge et les prêts importants consentis à sa famille font penser que le capitaine rapporta quelqu'argent du Canada. Il avança, en effet, à son père, 44 livres le 15 juillet 1669, 60 livres le 29 juillet suivant, 17 louis d'or le 28 août suivant, 1 louis d'or un autre jour, 14 livres $1 / 2$ représentant des perruques, le 10 février 1671, 22 livres le 10 mars 1671, 9 louis d'or, le 14 avril 1671. Sa mère bénéficia d'un prêt d'1 louis d'or le 15 mars 1670, un de ses frères eut 2 avances, 1 de 17 livres pour un justaucorps et 1 de 6 livres pour du plomb. Les éléments de comparaison portant sur le coût de la vie en France et au Canada sont assez vagues. Compte tenu des vivres accordés par le roi durant le séjour à la colonie, le capitaine semblerait y avoir touché une solde supérieure d'environ $50 \%$ s'il avait été logé gratuitement à Québec, ce qui ne semble pas correspondre à la réalité. En estimant le logement à la valeur d'un repas, il aurait gagné environ $1 / 3$ de plus à Québec qu'à Bordeaux. Compte tenu de la réévaluation canadienne d'environ $1 / 3$ avec prime la portant à la moitié pour le louis d'or, ${ }^{14}$ les 49 louis tenus par la logeuse, la demoiselle Amiot, représentaient environ 700 livres argent de France pour 14 mois, soit 50 livres par mois et 30 sols par jour, dépenses paraissant proportionnée avec 20 sols d'auberge +3 sols de couchage payés à Bordeaux, étant vraisemblable que la demoiselle Amiot nourrissait de Tapie. Comparativement avec la vie moderne, la solde du capitaine doit

14 Adam Shortt, Documents relatifs à la monnaie, au change et aux finances du Canada (Ottawa, 1925), $8^{\circ} \mathrm{Pa} 230$ (I), à la Bib. Nat. 
être comparée au minimum à celle d'un commandant, car le grade de capitaine était alors important. Les 900 livres annuelles dont était gratifié de Tapie représentaient donc au moins 1800000 fcs et la livre environ $2000 \mathrm{fcs}$. Cette évaluation correspond aux 20 sols par jour à l'auberge de Bordeaux et au prix actuel de $2000 \mathrm{fcs}$ pour une pension modeste et mensuelle de $2000 \mathrm{fcs}$ par jour sans logement, compte tenu de ce que la nourriture devait être beaucoup plus abondante au XVII ${ }^{\mathrm{e}}$ siècle..$^{15}$ Les 6 couteaux au Canada représenteraient aussi 2000 fcs, soit plus de $330 \mathrm{fcs}$ par instrument tranchant. Le fusil à 20 livres, monnaie de Canada, représenterait $53000 \mathrm{fcs}$, frais de transport compris, ce qui est seulement un peu cher. Le vin et l'eau-de-vie ne peuvent être appréciés, faute de connaître la contenance des barriques. Par contre, même avec de la dentelle de Valenciennes et une bonne doublure, le gilet de baraguan à 54000 fcs paraît exhorbitant. Les dépenses de 6 livres, soit $12000 \mathrm{fes}$ pour du plomb de chasse, de 126 livres, soit 252000 fes pour du linge, sont hors de proportion avec les conditions de la vie moderne. La valeur des denrées alimentaires semble, par contre, être demeurée à peu près stable, compte tenu de celle de la monnaie.

ROBERT LE BLANT, 24 rue de Varize,

Paris XVI.

(à suivre)

15 Nous avons évalué en 1957. 